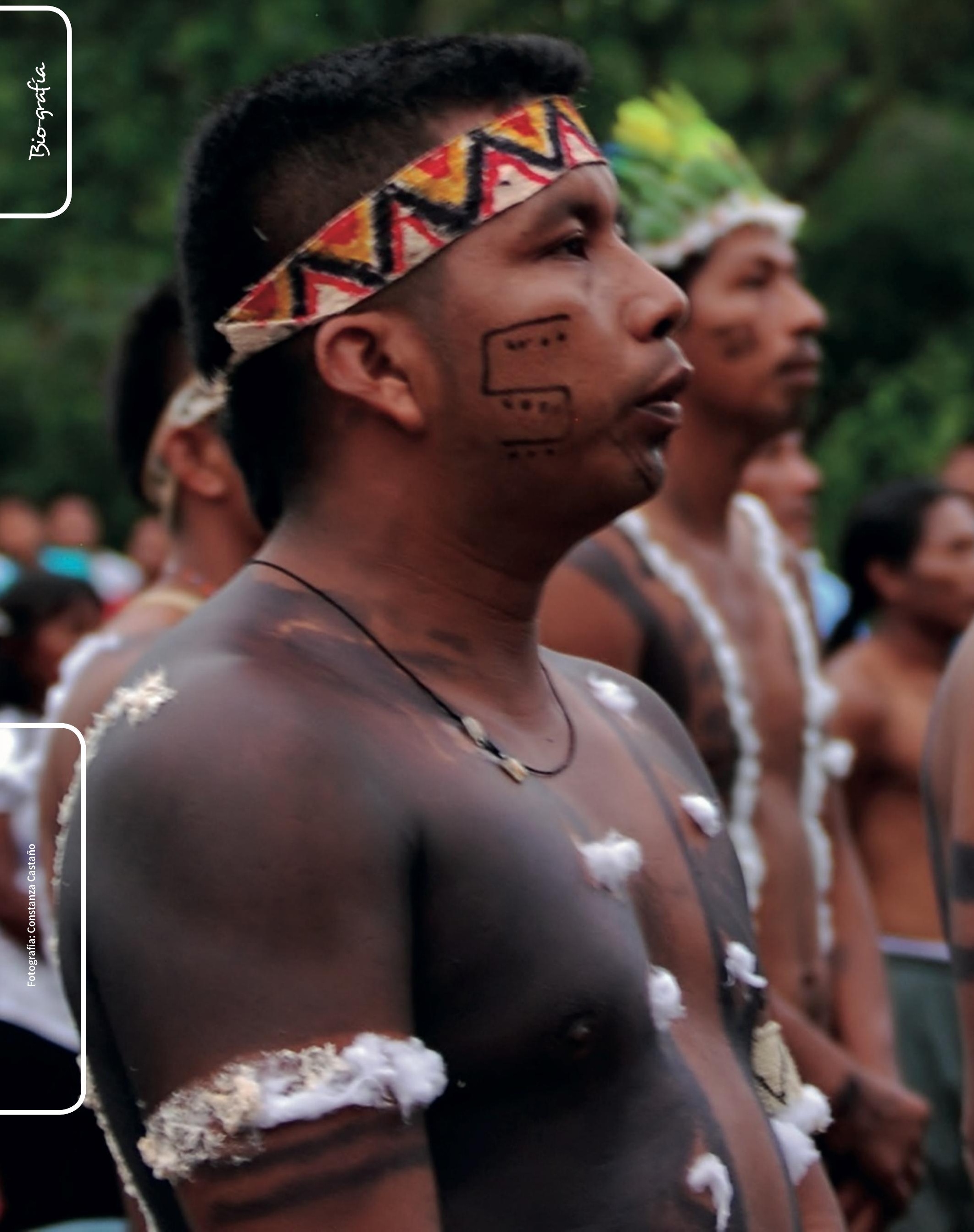




\section{SABERESTRADICIONALES SOBRE EL USO Y MANEJO DEL P+CAÁJKE EN LA ETNIA BORA, COMUNIDAD DE PROVIDENCIA Y SU RELACIÓN CON LA EDUCACIÓN PROPIA}

Fecha de recepción: 25 de septiembre de 2013 Fecha de aprobación: 13 de diciembre de 2013

\section{Josefina Teteye Eimenekene ${ }^{1}$ José Fernando Teteye ${ }^{2}$ Jesús Ángel Teteye ${ }^{3}$ Norma Constanza Castaño Cuéllar ${ }^{4}$}

\section{Resumen}

Investigación realizada en el río Igaraparaná, Amazonia colombiana, con la comunidad indígena bora de Providencia, en los clanes Ts+ts+vemuna, Boaj+, +vamuje. El propósito fue documentar los saberes tradicionales en torno al significado, uso y manejo del p+caájke y realizar una descripción etnobotánica de la planta.

La metodología es de corte hermenéutico, con una técnica etnográfica, que implicó fases de descripción, categorización e interpretación.

Como resultado se encontró que los saberes, uso y

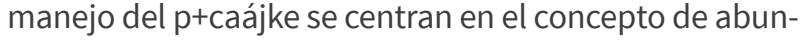
dancia, constituido por ocho dimensiones relacionadas con la prevención de males, mayor producción de los frutos de la chagra, valores femeninos, trabajo, alimento, espiritualidad, salud, armonía y variedad.
Con base en esta caracterización, se realizó una descripción de los procesos educativos propios de la etnia bora y se hizo una propuesta pedagógica, de carácter intercultural, que busca generar un diálogo de saberes, entre los conocimientos ancestrales en torno al p+caájke y sus significados, con el conocimiento escolar, específicamente en el grado tercero de primaria.

\section{Palabras clave}

Comunidad bora, saberes tradicionales, uso y manejo p+caájke, concepto de abundancia, pedagogía en contexto.

1. Nombre tradicional "I+ye", que traduce pepa de yuca, pertenece al clan Amanecer (Ts++ts+vemúnaa) del pueblo bora. Licenciada en biología, Universidad Pedagógica Nacional, Ceres, La Chorrera (Amazonas). joteteye@gmail.com

2. Anciano del clan Amanecer (Ts++ts+vemúnaa), pueblo bora.

3. Sabedor tradicional clan Amanecer (Ts++ts+vemúnaa), pueblo bora.

4. Docente Universidad Pedagógica Nacional, Departamento de Biología. Coordinadora Grupo de Investigación Enseñanza de la Biología y Diversidad Cultural. Estudiante Doctorado en Educación. Sede Universidad Distrital Francisco José de Caldas. constanza_castano@yahoo.es 


\section{Abstract}

Investigation performed at the river Igaraparaná, Colombian Amazonia, with the indigenous community of Providence Borain the clans Ts+ts+vemuna, Boaj+, +vamuje. The purpose was to document the traditional knowledge about the meaning, use and management of p+caájke and a ethnobotany description.

The methodology is hermeneutic cutting with an ethnographic technique, which involved phases, description, categorization and interpretation.

As a result it was found that the knowledge, use and management of p+cajke, focus on the concept of abundance, consisting of eight dimensions: related to: the prevention of injury, increased production of the fruits of chagra, feminine values, work, food, spirituality, health, harmony and variety.

Based on this characterization, make a description of the educational processes of ethnic Bora and educational approach, with intercultural character, which seeks to create a dialogue of knowledge, among the ancient knowledge about the p+caájke and their meanings, with school knowledge, specifically in the third grade level.

\section{Key words}

Community Bora, traditional knowledge, use and management $\mathrm{p}+\mathrm{caájke}$, concept of abundance, education in context.

El pueblo bora está ubicado en el corregimiento de La Chorrera, Departamento del Amazonas (Colombia), sobre la ribera del río Igaraparaná. Es uno de los grupos étnicos que lograron sobrevivir a la barbarie del caucho, y que ahora están comprometidos en la reconstrucción social de su cultura, consolidando su identidad, autonomía y territorio.

Las prácticas culturales del pueblo bora se vieron afectados por el proceso de poblamiento en el predio Putumayo desde el comienzo del siglo XX, con la intervención de la Casa Arana en la explotación del caucho, lo cual generó un genocidio y, en consecuencia, el desarraigo del conocimiento tradicional y de las formas propias de enseñanza que se impartían en los núcleos familiares de generación en generación a través de la tradición oral (Teteye R., 2012).
Esta investigación tiene en cuenta esta problemática, quees de vital importancia para la reconstrucción social y cultural de la etnia bora, e indagó por el significado, usos y manejo del p+caájke, en conjunto con los ancianos y jefes de clanes de la comunidad de Providencia, de los clanes Amanecer ${ }^{5}$ (Ts++ts+vemúnaa), Boa (Booánamu) y Guacamayo Rojo (++vaámuje). Se tuvieron en cuenta principalmente los conocimientos de los ancianos Meicuaco, Teteye y Evachiu.

El p+caájke es una planta sagrada, que se considera elemento espiritual en el pueblo bora, dado que simboliza la abundancia y se utiliza como tal en las prácticas culturales.

Dentro de la literatura que hay sobre el tema, encontramos que existe un fuerte énfasis en las investigaciones científicas sobre plantas en el Amazonas, pero ninguna hace referencia a conocimientos propios de la etnia bora $y$, específicamente, a estudios que tengan que ver con los usos y manejos de la planta p+caájke.

Se investigaron temas relacionados con la concepción del p+caájke desde el contexto cultural propio, conocimiento que en el momento no se ha tenido claro entre los pobladores de la comunidad, por la incursión del hombre blanco y de otros agentes externos a la cultura lo que de alguna forma ha cambiado ciertas prácticas culturales como los mitos, ritos, la protección de la chagra y las significaciones que se tejen en torno al p+caájke.

Para abordarlos temas sobre los usos, manejos y aplicaciones del p+caájke en contexto cultural, se desarrollaron actividades de campo en la chagra, como la caracterización de la planta y las formas de siembra y sus cuidados. El resto de la investigación se desarrolló directamente en los mambeaderos ${ }^{6}$, donde se interactuó con los ancianos, en lo relacionado con el mito de origen, sus enseñanzas, los usos y manejos de esta planta.

La metodología fue de tipo hermenéutico, etnografía como técnica y consideró espacios tradicionales del pueblo bora, como el mambeadero y la chagra. Se utilizaron entrevistas y conversaciones libres con ancianos, ancianas, mujeres y hombres adultos, al igual que con jóvenes.

5. Es el nombre que se le atribuye a un clan y simbólicamente representa a los animales que salen únicamente en la noche, como la boruga y la rana especialmente. El máximo representante de este clan es el anciano José Fernando Teteye.

6 Espacio donde se establecen los diálogos nocturnos, para narrar historias, realizar conjuros, dar consejos, y organizar el trabajo diario. 
La investigación se desarrolló bajo los enfoques de la línea de investigación Concepciones Acerca de la Vida y de lo Vivo, en contextos culturalmente diferenciados, de la Universidad Pedagógica Nacional, Departamento de Biología, que tiene entre sus propósitos contribuir a la comprensión de nuestras propias problemáticas educativas, especialmente las que se suscitan en torno a la formación y a la diversidad cultural.

Se concluye que el p+caájke, dentro de la etnia bora es considerado un vegetal sagrado, por ello tiene unas prácticas de cuidado específicas. El conocimiento tradicional, sobre el p+caájke es manejado diferencialmente por los ancianos de la comunidad y las mujeres. Los jóvenes en general desconocen los distintos aspectos relacionados con esta planta.

El conocimiento y las prácticas que se tejen en torno al p+caájke están centrados en el concepto de "abundancia" el cual tiene ocho dimensiones relacionadas con la prevención de males, mayor producción de los frutos de la chagra, valores femeninos, trabajo, alimento, espiritualidad, salud, armonía y variedad.

Dada la necesidad de recuperar estos conocimientos, especialmente con los niños y jóvenes, se hizo una descripción de los procesos de educación propia y una propuesta pedagógica, que busca generar un diálogo entre el conocimiento ancestral y el conocimiento escolar, que pretende compartir espacios de aprendizaje propios, como la chagra y la maloca; vincular a ancianos y a mujeres, haciendo las necesarias articulaciones con el proyecto educativo comunitario.

\section{Referentes conceptuales}

Entre el pueblo bora existen distintos conceptos que orientan el propósito de cuidar la vida. Entre ellos se destacan:

\section{Abundancia}

La abundancia se manifiesta en la mayor producción de especies y variedad de la siembra en la chagra, al igual que en la procreación de la humanidad.La abundancia es el resultado y cumplimiento de los consejos, dietas y sacrificios; es decir, para obtener cualquier tipo de beneficios llámese alimento u otro beneficio común, es necesario cumplir una serie de normas de prevención para garantizar el equilibrio del entorno, acompañado de la buena palabra, dedicación y amor hacia los demás.

\section{Chagra (uúm+e)}

La chagra es uno de los componentes de la vida, donde se enseña y se forma al ser humano en el trabajo agrícola y en valores.

En este lugar se siembran variedad de semillas como son los frutales de caímos, guamo, uva, mango y cacao; los tubérculos, plantas medicinales, para el sustento familiar, entre otros. De allí se abastece a la familia, se realizan las fiestas tradicionales, se curan las enfermedades y se saca la leña para preparar los alimentos.

Según Román 2005, citado en Donato, Escobar, Escobar, Pazmiño, y Ulloa (2007, p. 35):

La chagra es la consagración del pensamiento y la palabra en obra, es donde se materializa el saber, por lo tanto el alimento es sagrado y transforma el corazón y el pensamiento del ser humano, educa al ser humano, el trabajo educa al espíritu y educa la materia.

En la chagra se educa al espíritu y la materia; en ella está el secreto de recibir y mantener la sabiduría, "cada planta sembrada tiene un espíritu vivo, es alimento, curación, educación, canto, rito, matrimonio, bautizo, es la insignia de 'palabra y poder' de la pareja y de la familia” (Rufina, 2005; citada en Donato et al., 2007, p. 35-36).

Otros conceptos muestran que la chagra es "espacio de producción de insumo y alimento relacionados con la forma de vida y ejercicios rituales y sociales de las comunidades indígenas que en contacto con ella forman parte de un desarrollo y permanencia en el tiempo" (Vanegas, 2011, p. 33).

De acuerdo con Garzón, N. C. (en Leguizamo, s.f., p. 16):

[...] la chagra es el lugar más importante, [...] en el sentido de reproducción económica, es lo que le permite a esas sociedades mantenerse, tener un alimento de pan coger, contar con plantas medicinales pero, a la vez es un espacio donde se recrea la cultura, es un espacio para los estudios bioculturales, porque no solamente está la síntesis que tienen los indígenas del manejo del medio, sino también es la síntesis de la cultura, allí se ve los roles, el oficio de la mujer que trabaja ciertas plantas como la yuca, el ají, los frutales. 


\section{Mito de origen}

El mito de origen son principios y fundamentos para la orientación del manejo y práctica del calendario ecológico. Allí se manifiesta la creación del mundo, de los primeros seres de la humanidad y de lo que existe en el universo, por ejemplo; el origen delos clanes, de los grupos étnicos; el origen de cada una de las especies vivas que existe sobre la faz de la tierra.

De cada mito, se origina un comportamiento; es decir, una enseñanza para el ser humano, así mismo se dan las oraciones para la prevención de enfermedades, por eso es que los mitos son sagrados para la etnia bora. Según Román (citado en Donato, 2007, p. 35), "desde el origen todo fue ordenado con códigos, normas y leyes propias que fueron dadas a cada clan de acuerdo con su etnia".

\section{Saberes tradicionales}

Los saberes tradicionales son conocimientos que milenariamente se han venido manteniendo de generación en generación para desarrollar ciertas actividades dentro de la cultura; entre ellos está la forma de elaborar el mambe, el ambil ${ }^{7}$, de sembrar el tabaco, la coca; ${ }^{8}$ en los trabajos de la mujer está la forma de elaborar el casabe ${ }^{9}$, de sembrar maní, de elaborar la caguana ${ }^{10}$, entre otros trabajos representativos de la cultura.

Estos conocimientos se comparten con toda las criaturas que al final son la garantía de pervivencia de la humanidad, para preservar la diversidad de saberes para el fortalecimiento del pueblo y promoción de las expresiones culturales para el beneficio de las actuales y futuras generaciones.

Desde el punto de vista cultural, intelectual y espiritual los saberes tradicionales integran la vida social y cultural para el manejo de la vida.

7. Es el zumo de la nicotina del tabaco, que se obtiene después de cocinar la hoja del tabaco, exprimir la hoja y cocinar hasta obtener una masa negra.

8. Planta sagrada que el padre creador le otorgó al hombre bora para que realizara sus trabajos, cuidara a sus criaturas y este en relación con el mundo cosmogónico. Con la hoja de la coca se realiza el mambe, que consiste en tostar la hoja sobre una lata o tiesto de barro, luego machucarlo y cernir el polvo en una bolsa hecho de tela. El mambe se utiliza para realizar conjuros, organizar trabajo, ir de cacería y tener diálogos nocturnos.

9. Torta que se prepara con el almidón de la yuca y se asan sobre un tiesto. Es la comida típica de los bora, se prepara todo los días.

10. Bebida tradicional, que se prepara hirviendo el agua, luego el almidón de yuca, a esta bebida se le puede agregar diferentes sabores con frutas de la región.

\section{El mambeadero}

Escenario de construcción de conocimientos donde se practican los rituales e imparten el conocimiento y la sabiduría. También se considera un lugar apropiado para reunir a la comunidad para reflexionar e impartir consejos a los jóvenes o realizar trabajos en beneficio de la comunidad o de la familia.

\section{Hacer amanecer la palabra}

Para hacer amanecer la palabra es necesario plasmar las acciones bajo planes, programas y proyectos de vida; así las intenciones se convierten en obras: "Esta dimensión le corresponde a la persona como autoridad, para hacer realidad o hacer amanecer el pensamiento dicho por los ancianos" (azicatch, 2004, p. 88).

Es el beneficio hecho realidad, que recibe todas las criaturas de un gobierno que ama a sus criaturas y está representado por la juventud, la fuerza del progreso en la armonía universal. Estas competencias están definidas en los niveles de ancianos, autoridades, administradores y la población respectivamente. (azicatch, 2004, p. 88)

\section{El buen vivir}

El buen vivir es el respeto y cumplimiento de los mandatos de la ley de origen y el derecho mayor, es decir, la vida. Para ello es fundamental mantener el equilibrio "hombre-naturaleza", por ejemplo, teniendo una vivienda digna, buena chagra con buenos sembríos y en abundancia. Cuando se cumple, se vive bien y se obtiene el buen vivir.

Cuando existe la ruptura en los pensamientos no se logran los objetivos y se va en contra del mandato de la palabra de vida que dejó el padre Mepiivyéju Niimuhe, en relación con el cumplimiento del consejo y la dieta, para obtener buena calidad de vida/vivir bien.

Siendo así, el buen vivir se define como una política y estrategias fundamentales, relacionadas con la enseñanza, respeto, armonía y equilibrio con todo lo que existe, es decir, debe existir el respeto mutuo entre la naturaleza y el hombre para que haya armonía entre las partes.

\section{Pedagogía en el contexto}

En el pueblo bora una de las formas de enseñanza que más se utiliza para la transmisión de los conocimientos propios es a través de la oralidad: los ancianos trasmiten los conocimientos en los mambeaderos por medio de la palabra. 
La pedagogía propia, según Vanegas (2011, p. 27), hace referencia a la "recuperación de las formas de enseñanza que parten del propio contexto, sus fuentes de comunicación, las tradiciones entre otras".

Las comunidades indígenas, los campesinos, las negritudes han desarrollado durante siglos metodologías propias, autónomas, de trasmisión de conocimientos a las nuevas generaciones con un enriquecimiento adicional, fruto de la experiencia de los mayores y de mecanismos sociales de perfeccionamiento como son los ancianos, mayores indígenas, las parteras de las comunidades.

\section{Metodología}

La investigación es de tipo hermenéutico, como elemento fundamental para la interpretación y la comprensión del ser humano, por lo cual es necesario considerarlo en todas sus dimensiones y relaciones con el contexto cultural.

La investigación se realizó en los espacios tradicionales del pueblo bora, entre ellos principalmente el mambeadero, como espacio ritual, donde circula la palabra de conocimiento que orienta la preparación del ser y el cuerpo. Allí se reúnen los ancianos o jefes de clanes, que son las máximas autoridades tradicionales dentro de la comunidad. Se trabajó con los ancianos de los clanes Amanecer, José Fernando Teteye; del clan Boa, Santiago Meicuaco y del clan Guacamayo Rojo, Eugenio Evachiu.

El otro espacio de investigación fue la chagra como el espacio de práctica: es uno de los escenarios para el uso y manejo del $p+c a a ́ j k e$, en el que la mujer juega un papel fundamental en la responsabilidad de la siembra y cosecha, para el sostenimiento familiar.

Como instrumentos se utilizaron entrevistas y conversaciones libres, para establecer el grado de conocimiento que tienen sobre el uso y el manejo del p+caájke y su caracterización. Para lograr la información relacionada con el manejo y uso, se consultó a cinco grupos de personas: ancianos, ancianas, mujeres, hombres adultos y jóvenes, así:
1. Ancianos (3)
2. Ancianas (3)
3. Mujeres adultas (3)
4. Hombres adultos (3)
5. Jóvenes (4)

De igual manera, se realizó una matriz donde se plasmaron las preguntas y las respuestas, desde las diferentes concepciones. Las actividades se desarrollaron en coordinación previa con autoridades locales de la comunidad: comuneros afiliados al cabildo bora de Providencia, jefes de clanes, institución educativa.

Las fases que emplearon durante el proceso de la investigación, corresponden a la descripción, categorización e interpretación.

\section{Resultados}

\section{Creencias y espiritualidad}

En la comunidad bora se practican los rezos o conjuros realizados por los ancianos para curar las enfermedades, mediante la creencia de los tres elementos que dejó el padre creador: tabaco, coca y yuca dulce, por medio de los cuales se invoca al espíritu de vida. La espiritualidad se mantiene de acuerdo a la relación hombre- naturaleza-seres espirituales. De igual manera se cree en los mitos de origen para cuidar los hijos y la vida social.

Los siguientes son los elementos importantes para vivenciar las creencias y la espiritualidad:

La coca: Planta sagrada, es el eje que orienta la palabra y dirige la comunidad.

Tabaco: Planta sagrada que representa la sabiduría del hombre. Por otro lado, el tabaco de la mujer, es decir, la sabiduría se materializa en el ají.

La maloca: Espacio sagrado de comunicación con los espíritus de nuestros antepasados y dioses.

El cacique: Jerarquía natural que viene de generación en generación cuyo título se otorga en el baile tradicional ante los ancianos y comunidad bajo un rito especial.

\section{Descripción etnobotánica del p+caájke}

El p+caájke se encuentra en el Departamento del Amazonas, en la jurisdicción del corregimiento de La Chorrera, dentro del pueblo bora. La forma de la siembra y cuidado es similar a la de la yuca (Manihot Esculenta), la cual se siembra por medio de estaca máximo de $20 \mathrm{~cm}$.

Para la siembra es importante seleccionar la semilla antes de sembrar que se puede hacer de dos formas: por medio de estacas y a través del tubérculo que es la misma raíz del p+caájke. 
Una vez obtenida y seleccionada la semilla, se introduce directamente sobre la tierra, en la hoguera que ya ha sido preparada.

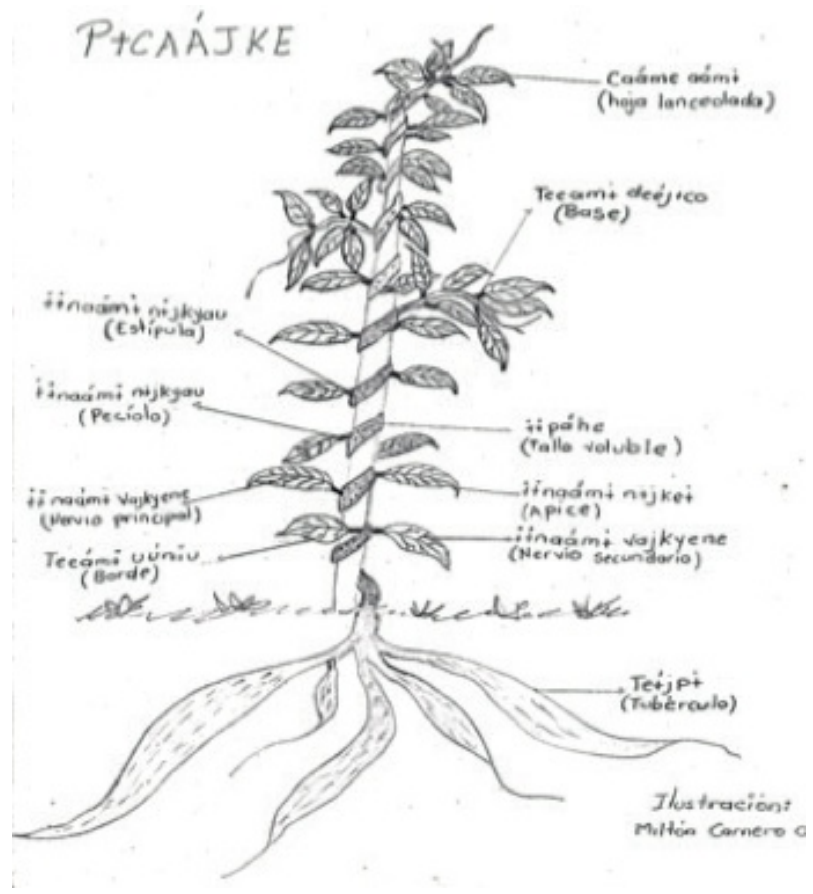

Figura 1. El p+caájke

Fuente: Ilustración de Milton Carnero.

En la siembra existe una diferencia, específicamente en el tiempo en que se va sembrar este bejuco; por ejemplo para el clan Amanecer (Ts+ts+vemúnaa) el p+caájke se siembra antes de sembrar otros tipos de semilla, para que ella sea la primera en crecer y pueda brindarle fertilidad a las demás semillas, como el ejemplo de una madre. El clan Boa (boaaj+) lo siembra después de haber sembrado las demás semillas, con el fin de no tocar más la chagra.

En cuanto al mantenimiento del p+caájke, como de la chagra, se realizan periódicamente limpiezas de las malezas, que pueden ser a través de mingas o simplemente a cargo de la familia. Sin olvidar que es necesario realizar hogueras con las malezas que se van eliminando porque la siembra que está en la chagra necesita humo biológico para su buen desarrollo.

Las características son las siguientes:

- Nombre común: P+caájke (en bora).

- Departamento del Amazonas, jurisdicción del corregimiento de La Chorrera, dentro del pueblo bora.

- Vaarícyo (es trepadora).

- ++páhe (tallos volubles, leñosos y gruesos).
- Llaraiíwa (mide 3 metros de altura).

- Te+jpe (tubérculos muy desarrollados y fuertes).

- Mohoú oonaba (corteza externa de color gris o blanco).

- Caáme aám+ (hojas lanceoladas y opuestas).

- ++naám+n+jkyau (sin estípula).

- ++naám+n+jkye+ (ápice acuminado).

- ++naám+n+jkyau (pecíolos delgados).

- Teéam+ uúniu (márgenes crenulados).

- Teéam+deéj+co (base obtuso).

- 8 a 10 pares de nervios secundarios.

- Del desprendimiento de ramas y hojas sale un exudado de color blanco.

- Del desprendimiento de cualquier rama u hoja de la planta sale un exudado de color blanco.

El p+caájke por ser un vegetal sagrado tiene algunas prohibiciones en cuanto al manejo y la utilización en las actividades culturales, como abstenerse de comer pescado que tenga aguijones, uno porque destruye al vegetal y otro porque los beneficios espirituales dejan de hacer efecto.

Otro de los secretos es la utilización de la masa del p+caájke antes de iniciar las labores de preparación de una fiesta tradicional, que consiste en untar la masa del $\mathrm{p}+$ caájke en toda las vasijas que se van a utilizar durante la preparación de la comida para la fiesta tradicional.

\section{Manejo y uso}

\section{Origen}

De acuerdo a las respuestas obtenidas de los ancianos se confirma que el p+caájke tiene un origen femenino, de uno de los vellos que fue arrancado de la cara de Cacameéwa. Esta respuesta se obtuvo una vez los ancianos terminaron de narrar el mito de origen.

De acuerdo al diálogo sostenido con los ancianos se evidenció que este vegetal representa a la mujer juiciosa o también conocida como la dueña de la abundancia. Se le atribuye este nombre porque de acuerdo al mito de origen se dice que la función de esta planta es garantizarle un desarrollo eficaz a todo cultivo que se siembra en la chagra, es decir que con el calor de este vegetal los frutales crecen robustos.

Los hombres adultos plantean que el bejuco tiene que ver con la abundancia, porque desde el mismo origen el dios creador encargó a este ser espiritual que trajera a este mundo todo lo relacionado con el trabajo y la comida en abundancia para el ser humano. 
Las ancianas mencionan que este bejuco se relaciona con la abundancia porque su mismo nombre traduce que es una mujer dueña del trabajo. Con ella se originan los tabúes para hacer rendir los trabajos de la mujer bora.

Por parte de los jóvenes no hubo respuesta alguna frente a este tema, porque sus padres no le han comentado sobre este mito de origen, únicamente estas historias se comentan en los diálogos nocturnos, espacio donde les queda muy difícil asistir.

\section{Beneficios}

Se confirma por las respuestas dadas por los ancianos que el aporte que hace el p+caájke al trabajo que desempeña la mujer y al desarrollo de las prácticas culturales, especialmente se refieren al trabajo de la chagra, el cuidado y mantenimiento para obtener la cosecha de los cultivos que allí se siembra.

\section{¿Quién es Cacameéwa?}

De acuerdo al diálogo que se sostuvo con las ancianas, Cacameéwa es el personaje mítico que representa a la mujer trabajadora, con ella se originaron los valores y el arte de ser buena madre. Ella es el ejemplo que las mujeres boras deben seguir

La respuesta de los adultos mayores corrobora las respuestas que dieron las ancianas. Ellos mencionaban que Cacameéwa representa a una mujer trabajadora, responsable, respetuosa y servicial. Ella dejó unos ejemplos de cumplimiento de los trabajos y el buen comportamiento, en especial el amor hacia el trabajo

Los ancianos mencionan que es de gran importancia narrar el mito de origen antes realizar el baile tradicional, porque a través del mito se invoca al espíritu y al aire de la abundancia para que se instaure y se materialice en los trabajos que se van a desarrollar antes de la fiesta.

Otra de las particularidades de narrar este mito se hace para contribuir con el trabajo de las mujeres con el rendimiento y educación de las criaturas; por lo que allí están los lineamientos de trabajo y comportamiento.

\section{Siembra}

Esta pregunta únicamente se realizó al grupo de ancianos, puesto que son ellos en quienes se han depositado los conocimientos de vida, para ser transmitidos.
Los ancianos mencionaron que las normas para la siembra del p+caájke están en el mismo mito de origen. En él se menciona que esta planta se debe sembrar en las hogueras y en la mitad de la chagra para que desde este lugar cuide y fortalezca a los demás cultivos.

De acuerdo a la respuesta obtenida del anciano José Fernando Teteye, se entiende que este vegetal se puede utilizar de dos maneras: como tangible, en las prácticas que se desarrollan en la chagra, y como intangible, es lo espiritual antes de iniciar un baile tradicional, para dar la acción de gracias al espíritu en donde se resalta el agradecimiento, diálogo permanente con la madre abundancia.

Desde el mismo mito, esta planta simboliza a la mujer dueña del trabajo y abundancia, por lo tanto, son las mujeres quienes se encargan de mantener y cuidar esta planta. La anciana menciona que el p+caájke se siembra en cualquier terreno esto es de menor importancia, porque el destino es donde se haga la chagra; puede ser sembrada en estaca o bulbo, con dedicación y amor como el fruto de abundancia que brindará fortalezas en la producción de alimentos.

De acuerdo a la entrevista realizada a la señora Zenaida, este vegetal se siembra removiendo el suelo, como el símbolo de amor hacia ella, esto se debe sembrar al lado de un palo para que ella pueda intervenir en su crecimiento dándole apoyo. Además, nos comenta que esta práctica siempre se ha mantenido para sembrar cualquier tipo de cultivo.

La anciana Emma Eimenekene manifiesta que el tiempo en que se debe sembrar el p+caájke es antes de sembrar las otras semillas. Después de que siembra el resto de semillas, esta planta le da el recibimiento con el aire de abundancia y continúa con el objetivo de "cuidado y protección”.

Manifiesta Zenaida Teteye que para contribuir en el buen crecimiento del p+caájke se deben realizar jornadas de mingas para que con el humo de las hogueras se alimente el p+caájke.

\section{Reflexión general}

Los resultados muestran que el p+caájke desde la tradición tiene un origen femenino, porque nació de uno de los vellos de la cara de la mujer (Cacameéwa). Dentro del mito de Cacameéwa, se originan una serie de valores que tienen que ver con los comportamientos sociales y de trabajo. Estos valores se han inculcado dentro de los 
núcleos familiares por cuenta de los ancianos, ancianas y padres de familia. En lo que se refiere al mito de origen, la mayoría de los entrevistados manifestaron que este conocimiento se ha venido difundiendo de generación en generación a través de la tradición oral, en el mambeadero.

Respecto al tema relacionado con el cuidado, uso y manejo del p+caájke; los cinco grupos concuerdan con las mismas respuestas, a excepción del grupo de los jóvenes, quienes dijeron que no tenían conocimiento sobre este tema, en razón al desinterés por acercarse a los espacios donde se imparten estos conocimientos.

Los resultados de este trabajo aluden principalmente a la abundancia, porque dentro de la narración mitológica está consagrado que allí se originó el secreto de mantener los alimentos continuamente en los núcleos familiares y se realizan actividades de aprovechamiento de manera comunitaria a través de las fiestas tradicionales. Además, dentro del mito se especifica que este vegetal simboliza la parte femenina, dueña del trabajo y llena de valores.

Otro de los aspectos que se resaltó fue el manejo espiritual que se le da a este vegetal durante la realización de las fiestas tradicionales, en donde la mayoría de los ancianos decían que lo espiritual se maneja desde el mambeadero, que es el espacio indicado para invocar al espíritu que se personifica en Cacameéwa o "Madre de abundancia", para que se manifieste en obra y los trabajos se desarrollen sin inconveniente.

De acuerdo a los análisis y reflexiones se consolidaron pilares fundamentales como el resultado de los diálogos sostenidos con los ancianos, ancianas dueñas de maloca, mujeres adultas y hombres adultos. Desde allí, se resaltan ocho pilares que aluden a la abundancia:

1. Previene los males contra los sembríos.

2. Mayor producción de los frutos de la chagra y el robustecimiento de las siembras.

3. Valores; define el rol de la mujer bora, como trabajadora, servicial y respetuosa.

4. Trabajo; propicia el ánimo en las labores de chagra y tiene implicaciones espirituales por lo que en ella están todos los lineamientos de protección.

5. Alimento; por lo que se cuenta con la base de subsistencia segura para la contribución al buen vivir.

6. Espiritual; porque se relaciona desde lo tangible cuando se siembra en la chagra y se extrae la masa para untarse las manos de las manipuladoras. Y en lo segundo, desde lo intangible, cuando se invoca al espíritu, al inicio de las acti- vidades desde el mambeadero, para encontrar rendimiento en la comida (el almidón, el maní, la mafafa, etc.) durante la fiesta.

7. 7. Salud; por lo que contribuye en el cuidado y protección de la salud de la mujer y de la siembra; es así como obliga a una serie de dietas para que se dé efectividad del mandato divino, "por lo que es una madre que cuida a sus hijos".

8. 8. Armonía y variedad; teniendo en cuenta que la chagra es el factor de múltiples variedades de siembras, donde se amanece la palabra para la realización de diferentes actividades familiares y comunitarias. Es decir, aportando a construir el buen vivir, con armonía y variedad.

Estos resultados enriquecen el concepto de la palabra de abundancia, la cual desde la cultura bora se define como:

- La base del desarrollo propio.

- La adquisición de sabiduría, con el fin de conocer las diferentes especies y variedades de semillas, para transmitir los saberes a las criaturas.

- El resultado de escuchar los consejos y el cumplimiento de las dietas, para la obtención de buenos resultados en los sembríos y el control de las plagas y malezas.

- Tener buena salud, convivencia y armonía: "Cuando hay cosecha hay buena salud".

Por lo anterior, es preciso resaltar que en la abundancia está la sabiduría y la transmisión de saberes. El éxito de la cual se logra en la clasificación de las semillas, por lo que se define como palabra de abundancia, que alude a los ocho pilares mencionados en el concepto de abundancia, como el aporte a la palabra.

Dentro de la entrevista se tuvo la oportunidad de dar participación a los jóvenes, lo que arrojó un alto grado de desconocimiento de este tema, por ello se diseña una propuesta pedagógica en aras de involucrar este conocimiento de vital importancia dentro del aula escolar.

Igualmente, es preciso resaltar del proceso investigativo los aspectos relativos a la formación como mujer bora, ${ }^{11}$ los conocimientos propios transmitidos y retroalimentados por medio de las prácticas en los diferentes espacios de conocimiento propio y occidental: la escuela, el mambeadero y la chagra. Además, los valores que se

11. Específicamente se refiere aquí a la experiencia de Josefina Teteyé como mujer bora y en el transcurso de su formación como licenciada en biología de la Universidad Pedagógica Nacional en La Chorrera, Amazonas. 
le atribuyen a la mujer bora por lo que desempeña un papel fundamental en el sostenimiento de la cultura y la responsabilidad en los diferentes aspectos de la vida de la comunidad.

Como criatura bora igualmente es necesario resaltar el conocimiento de los mayores, es decir, la base tradicional a partir de la cual se construyen los conocimientos. Al haber realizado esta investigación se conoce, se siente y se tiene la capacidad de desenvolverse como profesional de la enseñanza, mostrando la importancia del conocimiento propio a las nuevas generaciones. Además, después de la investigación, surge el compromiso de dar respuesta al mandato encomendado por los mayores, en la transmisión de las enseñanzas a las criaturas y especialmente la valoración del género. El ejercicio investigativo permitió ampliar las formas de ser y hacer conocimiento en dos escenarios de aprendizaje "mambeadero-chagra" que se destacan porque desde allí se imparten los conocimientos para la formación propia.

En cuanto a los conocimientos adquiridos en la formación como licenciada en biología, se considera que los conocimientos adquiridos complementaron la formación tradicional y contribuyeron a rescatar y valorar lo propio. Es así como se aprendió a interactuar con el entorno, teniendo en cuenta que todo lo que nos rodea es vivo y como tal debe ser valorado y respetado. De acuerdo a la formación intercultural recibida, con estos conocimientos es posible desenvolverse en los diferentes contextos, con el fin de aportar en el direccionamiento de los procesos comunitarios e institucionales.

Reconociendo y valorando las vivencias y principios de educación propia de los clanes Ts++ts+vemúnaa, ++vaámuje y Booánamu, se resalta que la educación de la criatura bora comienza desde el vientre, por lo cual es hereditaria culturalmente.

El proceso de construcción de conocimientos en el mambeadero surge a través de los diálogos nocturnos dependiendo del calendario ecológico. La educación es una orientación de vida que se da en el mambeadero, a través de los mitos de origen mediante comparaciones de los personajes míticos, de las cuales surgen los consejos para la orientación de la vida y que requiere de dietas para ser personas formales y de bien, con los principios de servir, ayudar, amar al otro.

La educación se manifiesta igualmente por medio del trabajo de los padres hacia los hijos, de acuerdo a las diferentes especialidades para el desenvolvimiento en la vida cotidiana. Está ligada a la identidad, la cultura, los valores humanos y la espiritualidad, como los principios de vida de la criatura bora que surge de acuerdo al pensamiento, palabra y obra.

En el segundo escenario donde se imparten los conocimientos de formación propia se visibiliza la chagra, que surge a través de actividades de socola, tumba y quema. Donde se transmite el conocimiento papá-hijo para que se forme y contribuya con el bienestar familiar y social. Posteriormente, se transmite la enseñanza de madre-hija en la siembra. En la cosecha, la enseñanza es mutua padres e hijos, para el debido mantenimiento en el futuro del banco de alimento ${ }^{12}$ y sabiduría. Por último, la transmisión de saberes se consolida en la cosecha, como el resultado de la orientación y enseñanza, es decir: "palabra-obra".

De esta manera se consolidan los conocimientos propios en colectividad, pues es colectivamente que se logran los procesos desde estos escenarios de aprendizaje "mambeadero-chagra".

En este contexto educativo y de formación, surge la siguiente propuesta pedagógica, que implica narrar el mito de origen del p+caájke a los estudiantes del grado tercero, empleando mecanismos propios de enseñanza, para que conozcan la importancia de este vegetal y se concienticen con la enseñanza que deja el mito.

Como metodología de trabajo, se proponen una serie de actividades para motivar el interés por aprender los saberes culturales que se tejen en torno al mito de origen:

Llevar a los estudiantes a cualquiera de las seis malocas que existen en la comunidad de Providencia, para que el anciano les narre el mito de origen, a través de la siguiente metodología:

1. Dramatizar el mito, dando participación a todos los estudiantes, empleando elementos propios del medio.

2. Formar dos grupos de trabajo, donde las niñas se reúna con la ancianas y los niños con el anciano, para que les inculque los valores que deja el mito, el significado que representa y el rol que deben desempeñar cada uno en el campo social.

3. Desarrollar actividades de campo, en coordinación con los dos ancianos, donde se practique las formas de siembra y cuidado del p+caájke.

12. Es el nombre que se atribuye a la palabra que maneja cada anciano; es decir, la palabra que le orienta y a la cual le rinden devoción. 


\section{Actividades a desarrollar}

1. Realizar actividades de campo de forma práctica para que haya participación de todos los estudiantes y así se facilite el entendimiento sobre los usos y manejo de la planta.

2. Realizar un comentario general sobre la importancia y uso de la planta.

\section{Competencias}

1. Conoce y maneja la importancia del mito de origen.

2. Desarrolla actividades en pro de la recreación de las plantas que están tendiendo a desaparecer incluyendo el p+caájke.

3. Practica lo aprendido con los dos ancianos.

4. Se interesa por asistir a los espacios donde se transmiten los conocimientos tradicionales de forma oral y práctica (mambeadero-chagra).

5. Demuestra interés por valorar el conocimiento propio.

6. Practica las enseñanzas que deja el mito.

En cuanto al enfoque pedagógico, se emplearán temas relacionados con la formación integral del ser humano, por ser un componente que se relaciona con la vivencia del ser humano. Las actividades académicas se enfocarán desde un punto de vista intercultural, aportando el conocimiento y las prácticas de enseñanza propias, con respecto a la didáctica y al currículo escolar. Es decir, se emplearán elementos propios de la región para motivar el trabajo en el aula de clases, sobre todo en las actividades lúdicas.

\section{Conclusiones}

El conocimiento tradicional sobre el uso y manejo del p+caájke únicamente es manejado por los ancianos de la comunidad y es empleado solo cuando se va a realizar el rito del baile tradicional.

Las ancianas, en general las mujeres, son las directas responsables de la siembra, el cuidado y el mantenimiento de este vegetal, ya que espiritualmente representa a una mujer dueña del trabajo.

Debido a este significado, las mujeres lo cultivan en el centro de la chagra, en una hoguera y al lado de un palo grande. Para que desde allí ella cuide, proteja y les de vida a los demás sembríos que se siembran en la chagra. Además tiene la misión de prevenir todo tipo de plagas o animales que quieran atentar contra las siembras.
El p+caájke, dentro de la etnia bora, es considerado un vegetal sagrado, por ello tiene unas prácticas de cuidado muy específicas, incluyendo dietas y otros aspectos que no son aceptados socialmente para que la planta no se muera o suceda otra cosa desagradable.

En términos generales tiene estas prácticas específicas porque desde el origen tiene una formación como un ser humano (Cacameéwa, mujer trabajadora, dueña de la abundancia), porque como se explicaba, no es más que la palabra o el espíritu que se invoca durante el baile tradicional para que los alimentos rindan y alcance para todos.

En la chagra es sembrado para que de vida a las siembras y sus frutos sean robustos para abastecer a las familias.

Por lo anterior, todo el conocimiento y las prácticas que se tejen en torno al p+caájke están centradas en el concepto de la "abundancia" las cuales tiene ocho pilares que surgieron a raíz de la investigación.

Se formularon orientaciones pedagógicas para la enseñanza del mito de origen, con un carácter intercultural, en las que se articulan los conocimientos y prácticas propias, con la enseñanza formal, las didácticas y el currículo escolar.

\section{Referencias}

Azicatch (2004). Abuelo tabaco plan de vida y ordenamiento de los hijos de tabaco, coca y yuca dulce. La Chorrera.

Donato, L., Escobar, E., Escobar, P. Pazmiño, A. y Ulloa, A (EDS) (2007). Mujeres indígenas, territorialidad y biodiversidad en el contexto latinoamericano. Bogotá: Universidad Nacional de ColombiaFundación Natura de Colombia-Unión Mundial para la Naturaleza-UNODC-Oficina de las Naciones Unidas contra la Droga y el Delito.

Leguizamo, V. (s.f.). La chagra; un espacio de roles, aprendizajes y autoabastecimiento. Convenio Cifisan-pronata.

Teteye, R. (2012). Impacto del proceso de la caucheria en el territorio bora. Leticia, Amazonas. Medellín: Instituto Misionero de Antropología (ima).

Vanegas Cañón, E. A. (2011). La chagra en La Chorrera, recuperación de saberes tradicionales a partir de una experiencia pedagógica en contexto [Trabajo de grado especialización]. Universidad Pedagógica Nacional, Bogotá. 